Página inicial: 245 - Página Final: 254

Tipo de artículo: Reflexión derivada de investigación.

\title{
Desarrollo sostenible y vivienda digna como punto de progreso social.
}

\section{Sustainable Development and Decent Housing as a Point of Social \\ Progress.}

\author{
Por: Victor Hugo Vega Mendoza ${ }^{1}$ y Raúl Ruiz Canizales ${ }^{2}$
}

Recibido: Junio de 2016 Revisado: noviembre de 2016 Aceptado: noviembre de 2016

\section{Resumen.}

Se tiene como propósito evidenciar en qué medida el desarrollo sostenible es fundamental en la agenda de las nuevas políticas públicas que buscan un amplio alcance a largo plazo. La problemática global no es capaz de resolver los problemas a nivel local de un contexto altamente moderno y cambiante. La tendencia actual de "pérdida de calidad de vida" orilla a las ciudades a cambiar su perspectiva de progreso y así el bienestar dependerá directamente del desarrollo social que exista. Mediante un análisis de contexto se comparte una política de calidad de vida exitosa, pies la estética debe ser concientizada sin menospreciar su potencial al recuperar la zona. El resultado es que la percepción de crisis es inminente debido a los problemas asociados al crecimiento, para lo cual se han adoptado soluciones rápidas consistentes en el uso de los instrumentos de planificación física que solo convierten quejas en problemáticas especializadas. Se concluye que la ciudad moderna, como la conocemos, se encuentra en grave peligro de extinción gracias a su modus operandi, para lo cual la variable principal local debe solucionar el bienestar de la población sin menospreciar su entorno.

Palabras clave. Calidad de vida, ciudad, local, política, urbanización.

\begin{abstract}
.
The main purpose is to show the extent to which the sustainable development is critical in the agenda of new public policies that seek a wide scope, in the long run. The overall issue is unable to solve local problems within a modern and changing context. The current tendency of "loss of quality of life" forces cities to change their perspective of progress, and in that way, well-being will directly depend on the existing social development. Through a context analysis, a successful policy of quality of life is shared, since aesthetics must be made aware without underestimating their potential when it comes to recovering the zone. The result is that the perception of crisis is imminent due to the problems associated with growth. For this purpose, fast and consistent solutions in the use of instruments of physical planning have been adopted, which only turn complaints into specialized issues. It is concluded that the modern city, as we know it, is in serious danger, threatened with extinction thanks to its modus operandi, and in that regard, the local main variable must solve people's well-being, without underestimating their environment.
\end{abstract}

Key words. Quality of life, Urban Growth, Urbanization, and Social Development.

\footnotetext{
${ }^{1}$ Licenciado en antropología social por la Facultad de Filosofía de la Universidad Autónoma de Querétaro, México. cursa Maestría en Administración Pública Estatal y Municipal en la Universidad Autónoma de Querétaro, México. Miembro de "Bitácora Social-México". Contacto: victorhugo.vhvm@gmail.com

${ }^{2}$ Doctor en Derecho - Universidad Autónoma de Querétaro. Profesor investigador de Tiempo Completo. Miembro del Sistema Nacional de Investigadores (SNI) del Consejo Nacional de Ciencia y Tecnología (Conacyt). Jefe de Posgrado de la Facultad de Derecho (Universidad Autónoma de Querétaro, México) y Coordinador de la maestría en Ética aplicada y bioética de la Facultad de Derecho del Consejo Nacional de Ciencia y Tecnología (Conacyt). Contacto: raul.canizales@hotmail.com
} 


\section{Introducción.}

Las grandes ciudades, pueblos e incluso pequeños vecindarios no crecen de la noche a la mañana. Son en el mejor de los casos el resultado de una cautelosa planeación por ingenieros y diseñadores civiles, administradores de proyectos, arquitectos, planeadores ambientales y supervisores. La integración de estas disciplinas es conocida como desarrollo urbano. Es un sistema de expansión residencial que crea las ciudades y, en las que, a su vez, las zonas residenciales son el principal punto de interés. Este ocurre por expansión en las zonas no pobladas y/o en la renovación de las regiones en decadencia. Para efectos de lo que aquí se pretende se entiende el desarrollo urbano como un "crecimiento y calidad de los nuevos desarrollos inmobiliarios de la ciudad, así como el aumento en el bienestar derivado del crecimiento urbano, su planeación y el acceso al crédito para vivienda". (Governing Council de ONU-Habitat Consejo del Gobierno)

El concepto de desarrollo sustentable ha extendido a diversas esferas, tanto en lo económico como en lo social, incluso en la planeación educativa. En este sentido, es así como se hace presente el término de sustentabilidad del desarrollo urbano.

Con el concepto de desarrollo sostenible se ha querido lanzar un proyecto político, económico y cultural de amplio alcance que sea capaz de hacer coherentes las exigencias ambientales con las exigencias del desarrollo económico, desde el punto de vista a largo plazo. Así, de acuerdo con el núcleo semántico de la expresión "desarrollo sostenible" los intereses de las generaciones futuras son puestos explícitamente en primer plano junto con los haberes de las generaciones presentes.

Las razones por las que se debe enfrentar la problemática de la sostenibilidad del desarrollo de las ciudades, es debido a que la ciudad tiene un impacto relevante en la sustentabilidad global (por ejemplo, a través del efecto de las emisiones de $\mathrm{CO}, \mathrm{CO} 2$ y NOx por parte del tráfico vehicular sobre el llamado efecto invernadero), pero las mismas causas que ponen en peligro la sustentabilidad global impactan la sustentabilidad local; esto sugiere que es más eficiente enfrentarse a un mismo problema desde lo local y no a partir de un contexto globalizador, que vayamos de lo micro a lo macro en cuanto a la gestión de problemáticas. Como menciona Comagni (2005, pp. 1-19), “(...) la ciudad debe ser entendida desde el punto de vista como sede de la interacción social, la creatividad y el bienestar colectivo". Sin embargo, los procesos que dan origen al crecimiento desordenado e ilimitado que sufre la ciudad en periodos de despegue económico y de rápida industrialización, así como los procesos de urbanización difusa (metropolización- vale decir que, la metropolización es una noción forjada por extensión del término "metrópoli", etimológicamente, ciudad-madre, para designar a un proceso de transformación cualitativa, a la vez funcional y morfológico, de las ciudades muy grandes), los procesos de urbanización ciudad-campo y la problemática de movilidad y de consumo energético, hacen que hoy la ciudad sea insostenible y, consecuentemente, genera la posibilidad de que los gobiernos sean siempre insuficientes.

La metropolización es una noción forjada por extensión del término "metrópoli" (etimológicamente, ciudad - madre) para designar a un proceso de transformación cualitativa, a la vez funcional y morfológico, de las ciudades muy grandes.

\begin{tabular}{l|l|l|l|l|l|l} 
AGO.USB & Medellín - Colombia & Vol. 17 No. 1 & PP 1 - 323 & enero - junio & 2017 & ISSN: 16578031
\end{tabular} 
$\mathrm{Al}$ anterior conjunto de factores se adicionan las trasformaciones globales de la sociedad y la dificultad con que las políticas públicas se ocupan del problema de la insustentabilidad del desarrollo urbano de ciudades, los cuales deben ser tomados en cuenta, ya que son el reflejo de una insuficiente accesibilidad a los beneficios del ambiente urbano para algunos ciudadanos, gracias a la influencia sobre el funcionamiento global.

La eficiencia de la sustentabilidad del desarrollo urbano de ciudades, según Camagni (2005)

(...) alude a la sustentabilidad local en el sentido de ser valorada por sus efectos sobre las colectividades locales, respetando una restricción de no generación de contaminación insustentable sobre regiones cercanas y sabiendo que una ciudad encaminada en una dirección "localmente" sustentable es una ciudad que participa activamente en la reducción de los efectos globales negativos ( Pp. 163-199).

En este sentido, la variable principal es el bienestar de la población local a largo plazo, vinculada a la prosperidad de la misma ciudad. Dicho bienestar debe estar en función al bienestar económico, físico y ambiental, incluyendo la preservación (actual y futura) de los recursos naturales existentes en una localidad, de los cuales la población -que habita en ella-, se beneficiará de las externalidades positivas lo que generaría, por tanto, una mejora en su calidad de vida. En el caso de ampliación a la ciudad del paradigma de la sostenibilidad tenemos distintas propuestas de definición y de enfoque:

- El nivel de análisis (Camagni, 2005, Pp. 213-219): local, transborder, global. Esta propuesta totalmente subjetiva y de elección de un ámbito de investigación, es la del análisis de los efectos locales: la sostenibilidad es valorada por sus efectos sobre las colectividades locales, respetando una restricción de no generación de contaminaciones insostenibles sobre las regiones cercanas y sabiendo que una ciudad encaminada en una dirección localmente sostenible es una ciudad que participa activamente en la reducción de los efectos globales negativos.

- Las variables objetivo. En este enfoque la variable prioritaria no puede ser otra que el bienestar de la población local a largo plazo, vinculado a la prosperidad de la ciudad en cuanto tal. La ciudad es, en abstracto, un gran valor económico, social, cultural que en la realidad corre peligro de ser destruida por una serie de retroacciones negativas derivadas de su desarrollo espontaneo y de prevalecimiento de señales y decisiones a corto plazo.

- Para alcanzar el objetivo anterior, es necesario una integración entre los principios reguladores de los diferentes subsistemas. Eficiencia privada, equidad social, calidad de estética y equilibrio ecológico constituyen moldes reguladores y objetivos de intervención totalmente lícitos pero parciales y antitéticos, que no conducen a la sostenibilidad.

Es necesario alcanzar:

- Una eficiencia asignativa a largo plazo, mediante la internacionalización de los costos sociales y la construcción de un buen mercado que valore adecuadamente las ventajas futuras y no solo las inmediatas. 
- Una eficiencia distributiva, que permita al mayor número de ciudadanos disfrutar de los servicios de la ciudad, de las ventajas de la aglomeración, de la variedad de opiniones disponibles. No se trata de construir la ciudad de la igualdad, que no es ni condición necesaria ni suficiente para la sostenibilidad, ni la ciudad sin conflictos: más bien, la ciudad debe dar cabida a la diversidad, debe defenderla, integrarla y reproducirla, garantizando la ausencia de discriminaciones, la permeabilidad y la movilidad vertical de la población, la renovación de las elites, la más amplia accesibilidad a las oportunidades abiertas; la ciudad sostenible no es una ciudad sin conflictos, sino que es una ciudad que sabe gestionar los conflictos.

- Una equidad ambiental, en sentido intergeneracional: una vez más, no se trata, tanto o no solo, de disponer de valores ambientales, sino de garantizar el acceso y disfrute a todos los ciudadanos, presentes y futuros. El elemento de la equidad aparece no como valor abstracto, sino como valor vinculado al elemento ambiental, en dos sentidos principales: en primer lugar, porque muchas políticas ambientales pueden ser costosas e implicar sacrificios mayores para las clases menos favorecidas y, en segundo lugar, porque dada la característica de muchos bienes ambientales de estar localizados en el territorio, es posible un proceso de exclusión de algunos usuarios potenciales y de disfrute privado de sus servicios.

Dentro de este contexto, se busca un desarrollo urbano sustentable local que garantice un nivel no decreciente de bienestar a la población local a largo plazo, sin que comprometa las posibilidades de desarrollo de las áreas vecinas y que contribuya a la reducción de los efectos nocivos del desarrollo sobre el medio ambiente.

\section{La calidad de vida.}

Existe una difundida percepción de una crisis urbana, de una tendencia apremiante a la "pérdida de la calidad de vida" (percepción personal de un individuo de su situación en la vida, dentro del contexto cultural y valores con los que vive, y su relación con sus objetivos, expectativas e intereses en muchas ciudades, revirtiéndose así las tendencias de la primera mitad del siglo, cuando las ciudades crecieron en una atmósfera de optimismo.

Este concepto llega a inferir con el estado de salud, estilo de vida, la satisfacción con la vida, el estado mental y el bienestar. Por consiguiente, la calidad de vida está relacionada con condiciones y estilos de vida, con aspectos como la educación, salud, ambiente, aspectos socioculturales, económicos y de satisfacción personal. Remite a una evaluación que cada sujeto tiene de su propia vida. "La calidad de vida es el criterio a través del cual se juzga en qué medida las circunstancias de la vida aparecen como satisfactorias o insatisfactorias y necesitadas de mejoras" (Dorsch F, 1995). En sí, el peso radica en cuánta importancia le da el sujeto con respecto a su propia vivencia y a dos factores fundamentales para una mejor "calidad de vida":

Los objetivos bienestar material como: ingreso económico, vivienda, comunicación, urbanización, condiciones ambientales, educación y factores laborales. Estos factores comúnmente son asignados como materiales y de bienestar que cubren los niveles de satisfacción básicos cumpliendo las expectativas ya mencionadas. Por otro lado, contamos con los factores subjetivos como: laborales, satisfacciones (sentimientos), felicidad y estado de ánimo positivo. 
Así los estándares de bienestar dependerán del desarrollo de cada sociedad y van desde la necesidad de cubrir aspectos básicos hasta las orientadas a la realización personal, familiar y profesional del grupo social.

Analizar la calidad de vida de una sociedad significa analizar experiencias subjetivas de los individuos que la integran y la percepción que tienen de su existencia dentro de ella. Es importante entonces conocer cuáles son sus condiciones objetivas de vida y qué condiciones tienen para evaluar el grado de satisfacción que consiguen. Así, José Blanco (1997) dice "que la calidad de vida se modela de acuerdo a la confrontación permanente entre los componentes protectores y sus procesos destructivos (o de deterioro) para el desarrollo vital humano" (Pp. 21-28). Por otro lado, es también importante considerar los elementos intangibles que potencializan y condicionan la calidad de vida de las personas.

Centrándonos en el tema bienestar material la preservación del espacio público o entorno físico es fundamental para la medición de una "calidad de vida", ya que si se cae en su amplio concepto se entiende como un sistema de valores, estándares o perspectivas que varían de persona a persona y según la posición social dentro de la comunidad. Sarason (citado por Maya, 2004) identifica a la comunidad como aquella percepción de similitud con otros (o con el Otro), además del reconocimiento de la interdependencia con los demás y la voluntad de mantenerla, la reciprocidad y un sentimiento del que uno es parte de una estructura más amplia, estable y fiable.

De esta forma podemos consolidar la identificación de las personas con respecto al espacio donde ellos viven y, a su vez, el sentido de pertenecía abre las estructuras necesarias para demandar necesidades que abren las puertas a las participaciones institucionales, tal como lo menciona Hombrados-Mendieta (2011, pp. 97-126) “(...) es aquí donde interacciona la estructura informal a través de la solidaridad, el apoyo y la conexión emocional y la estructura formal a través de la acción de las instituciones".

La ciudad es considerada como la unidad básica donde se desarrollan las estructuras espaciales y dinámicas que inciden sobre el comportamiento individual y social, en donde el sentido de barrio cobra más importancia por considerarse el área donde el individuo establece sus redes (sentido de pertenencia) sociales y los habitantes construyen un "sentido de comunidad", el sentido de comunidad facilita las relaciones y la integración social, la identidad social y la adaptación a nuevas situaciones.

Dentro del barrio se puede determinar un ambiente físico que mida la calidad de vida existente según Altman y Wondersman (1987):

- La relación que existe con otros vecinos

- Cómo se presta el apoyo social

- Cómo se tratan con respecto a la sociedad donde viven

La falta de sentido de comunidad se puede relacionar positivamente con los problemas de salud en poblaciones (Ahern, Hendryx y Sddhartan: 1996, pp. 911-923) como:

- Migrantes 
- Personas mayores

- Jóvenes

- Trabajadores en situación de riesgo

La calidad de los espacios públicos está asociada al sentido de comunidad y a la calidad de vida, el sentido de comunidad aumenta con los años de residencia. Pero a mayor densidad poblacional influye negativamente en la calidad de vida, aunque en los últimos años este punto ha entrado en constantes debates y más aún el sentido económico el cual cuenta con tesis y antítesis con respecto a esta misma variable, argumentando que el dinero no es la solución para una mejorarla. Por ejemplo, para Francis Giles (2012, pp. 401-409) “(...) la calidad de vida y el sentido de comunidad se relacionan positivamente con la satisfacción del aspecto físico, con los espacios verdes disponibles y con la percepción de seguridad”. Esto nos demuestra que cuando se puede especular con respecto a un mayor sentido del espacio, (número de árboles y una distribución del barrio adecuada, por ejemplo) se cuenta con una mayor percepción de calidad de vida, por ende una mayor apreciación de progreso; pero esto sólo lo hace quedar en meras especulaciones que podrían considerarse como ficticias, sólo amortiguan y encubren la situación real que se vive en la cotidianeidad, como se dice en los albores públicos en nuestro país, "se cumple con la función de bomberos"; por ello se debe tener cuidado con las percepciones abiertas de la ciudadanía con respecto a la calidad de vida y sus hogares.

En tal virtud Fried \& Gleicher (1996) mencionan que

(...) el espacio físico inmediato que rodeaba la vivienda tenía un considerable significado como extensión del hogar, por lo cual aumentaba el sentido de pertenencia y este significado del área física es uno de los componentes principales de la razón de satisfacción (Pp. 305-315)

Aquí se encuentra otro punto determinante dentro de lo que mencionan Fried \& Gleicher, conforme a la percepción individual y grupal que se tiene en el barrio o comunidad, al crear espacios en comunitarios se cuenta con sentido de pertenecía y de identidad que refuerzan los lazos entre vecinos provocando una mayor percepción de calidad tanto económico, tecnológico y/o espacial como cultural social y personal.

Dockendorff \& Rodríguez (1996), artículo resumido y editado por Ambiente y Desarrollo a partir de la intervención del autor durante el $6^{\circ}$ Encuentro Científico sobre el Medio Ambiente, organizado por Cipma, Santiago (del 6-8 de enero de 1999), observan recientemente que, paradójicamente, junto al progreso tecnológico, económico, social y cultural se agudizan simultáneamente las externalidades negativas y la percepción de una crisis inminente, la cual "(...) no solamente anticipa panoramas muy negativos para la calidad de vida de sus habitantes sino, además, sus externalidades constituyen un freno al desarrollo". La principal fuente de insatisfacción es la falta de empleo (derivando en pobreza generacional), la que se manifiesta con preeminencia en las comunidades de bajo crecimiento. En las áreas metropolitanas (llámese Querétaro, México, por ejemplo) aparece como un factor de insatisfacción preponderante la delincuencia, seguida de cerca por los problemas asociados al crecimiento desmedido (carga vehicular, ruido, contaminación, excesos deconstruccion de casa-habitación) y desempleo.

Esta fragilidad social denota fundamentalmente una crisis de convivencia, que se manifiesta principalmente debido a la magnitud relativa de la población, así como por el déficit en sus servicios y a las deficiencias en su estructura. El restablecimiento de la confianza mutua entre los 
ciudadanos parece ser el objetivo de mayor magnitud, justamente por corresponder actualmente al más importante factor de insatisfacción. Parte del problema debe abordarse desde los instrumentos convencionales de planificación física, al enfatizar el sentido de barrio, comunidad o colonia, así como la identidad de las comunidades locales, la jerarquización de la vialidad para enfrentar la seguridad de los peatones y, entre otros, el desarrollo de espacios públicos que fomenten la convivencia. Pero el grueso del problema es de orden social, de desarrollo de formas de relación y de convivencia, cuya promoción ha desaparecido casi por completo al priorizar otras medidas, con la consiguiente orfandad institucional. Por otra parte, la inversión en vivienda e infraestructura urbana están aparentemente orientadas a la superación de la pobreza. Sin embargo, toman importancia creciente (en la percepción de los beneficiados) otros aspectos hoy descuidados tales como accesibilidad, pavimentación, servicios urbanos y los aspectos cualitativos del diseño urbano que confieren identidad.

La sola excepción de la falta de empleo es lo suficientemente poderosa como para motivar a los habitantes de una ciudad a abandonarla; pone de manifiesto otra situación, respecto de la cual existe evidencia empírica: las disfuncionalidades urbanas perceptibles por lo sentidos, que son capaces de motivar que la gente se mude a otro lugar de la ciudad. La mejor muestra parece ser el fenómeno de suburbanización. Estas tendencias, que siguen de cerca lo ocurrido hace un par de décadas en el mundo desarrollado, muestran que a medida que aumentan el ingreso y la disponibilidad de información, la población desarrolla una creciente intolerancia a los factores susceptibles de causar molestia a las personas. Además, es posible adelantar que esa intolerancia aumente y se sea cada vez más sofisticada, adquiriendo la capacidad de discriminar con detalle creciente más y más sustancias dañinas, así como otros aspectos con importancia sensorial tales como ruido, visibilidad y "vistas".

\section{El concepto o la idea de "vivienda digna".}

La vivienda digna la catalogaría en México como un fenómeno regional de exclusión social, el desarrollo sostenible a nivel de territorio requiere un análisis del contexto, de la zona objeto de intervención y de las causas que originan los desequilibrios sociales y un diseño de actuaciones que contemplen una estrategia conjunta de los aspectos sociales y económicos.

Esta estrategia conjunta debe partir de la necesidad de una intervención integral en la que es fundamental considerar el territorio como unidad operativa básica unido al concepto de hábitat, introduciendo con ello el acercamiento entre lo social, lo económico, lo urbanístico y el medio ambiente.

La necesidad de contemplar la intervención integral en los diversos ámbitos sectoriales (salud, empleo, vivienda, servicios sociales, infraestructuras, medio ambiente, etc.) implica la corresponsabilidad de todas las instituciones públicas y privadas del territorio. Para ello es fundamental una coordinación interinstitucional que aglutine en torno a una estrategia común a administraciones, empresas, sindicatos, ONGs y cualquier órgano de representación ciudadana, así como la de los propios afectados.

La vivienda digna y la ciudad son factores de integración y exclusión social, destacando que la causa esencial de la imposibilidad de acceder a una vivienda de esta naturaleza es, casi siempre, económica (falta de recursos y/o dificultades en acceder a los préstamos hipotecarios). 
La labor de la administración pública a través de las políticas gubernamentales es colaborar en la promoción de viviendas y suelo, en especial para viviendas para grupos sociales sin capacidad de ahorro y en condiciones vulnerables. En este sentido, estas actuaciones, que deben ser siempre integrales, deberán estar lideradas y/o protagonizadas fundamentalmente por la administración local, dada su "cercanía" a los ciudadanos.

En este sentido, según Manuel Porras (Subdirector General de Programas de Servicios Sociales Ministerio de Trabajo y Asuntos Sociales, España) los tipos más generalizados de demandantes de vivienda de integración son:

- Personas sin hogar.

- Personas alojadas en infravivienda.

- Personas con dificultades de acceso a primera vivienda (especialmente jóvenes).

Derivado de lo anterior, las propuestas de integración residencial deben considerar los diversos estados de exclusión según colectivos específicos y mantener a la "población atendida" en su hábitat; lo que requiere programas específicos.

En el marco de la vivienda sustentable y la política pública actual existen cuatro puntos a considerar:

- Aumentar el acceso al financiamiento de vivienda, particularmente a familias de bajos ingresos.

- Promover el desarrollo de la vivienda sustentable.

- Consolidar el sistema de la vivienda nacional por medio de mejoras a la gestión pública.

- Consolidar el apoyo del gobierno federal para el financiamiento de la vivienda sustentable para la población de bajos ingresos.

En el caso de México, se trata de un tema que no falta en la agenda y en la planeación sexenal de los gobernantes en turno; por ejemplo, se puede leer en el contenido del Plan Nacional de Desarrollo 2013-2018 (Gobierno de la República de México, 2013) y en el Programa Nacional de Vivienda (Gobierno de la República de México, 2014), el establecimiento de las bases hacia la consolidación de la vivienda sustentable en México. Para el logro de ello, se accionan dichas bases a través de la coordinación interinstitucional como la Secretaría de Desarrollo Agrario, Territorial y Urbano (SEDATU), quien tiene como objetivo principal la implementación de la Política Nacional de Vivienda mediante un conjunto de proyectos que procuran una vivienda digna para los mexicanos, pero en la que se priorizan tres elementos fundamentales: a) un entorno urbano sustentable, b) reducción del rezago de manera responsable, c) la proyección de la imagen de un México próspero con responsabilidad global. Queda pendiente el análisis final del impacto y logros de lo que en aquel Plan de Desarrollo se ha trazado en materia de vivienda digna y desarrollo sustentable.

Finalmente, dos variables que tarde o temprano tendrán que amalgamarse para la generación de posibilidades reales de acciones sustentables en esta materia. 


\section{Conclusiones.}

Los principales objetivos que debemos plantear, en efecto, es mejorar la calidad de vida de las urbes. Las ciudades, por ende, deben someterse al gran esfuerzo de una construcción integral y así consolidar capitales sustentables, sociales, equitativas e incluyentes. Se parte de un ámbito local a lo global y así observar que la ciudad deberá estar regida por los escenarios locales que la transformará en una ciudad con instrumentos de planeación suficientes para convertirla en participativa y activa en los efectos negativos globales. El principal reto es la capacidad de reorganización espacial de las principales actividades con enfoque de sustentabilidad, así como el bienestar de la población y, en este sentido, fomentar un desarrollo económico, físico y ambiental.

De esta forma, tanto el desarrollo espontáneo como la toma de malas decisiones administrativas a corto y mediano plazo, no solo cumplen la función de bomberos para extinguir el fuego, sino que poco a poco van condenando a la ciudad a su propia extinción en lugar de buscar su preservación con políticas a largo plazo que la vayan adaptando y cambiando con el paso de los años.

Los conceptos de estos proyectos crean comunidad, y no sólo se toma en cuenta el atractivo arquitectónico, sino la forma de potenciar la recuperación de una zona para beneficio y disfrute de sus habitantes y de todos quienes la visitan. Los conflictos dentro de las ciudades siempre existirán y eso debemos tenerlo claro siempre; a pesar de ello la buena administración no es aquella que sea capaz de resolver todos los problemas existentes, sino aquella que sea capaz de gestionarlos de forma eficiente, pues no hay que olvidar que, desde la perspectiva de la administración y de las políticas públicas, los gobiernos siempre serán insuficientes.

Derivado de ello, el reto es crear una capital equitativa en la que las personas ejerzan sus derechos en igualdad de condiciones y oportunidades, iniciar procesos de planificación y regeneración urbana integrada.

\section{Bibliografía.}

Ahern, M., Hendryx, M. S., \& Siddharthan, K. (1996). The importance of Sense of Community on people's perceptions of their health-care experiences. Medical Care, 34, 911-923.

Altman, I., \& Wandersman, A. E. (1987). Neighborhood and community environments. New York: Plenum Press.

Blanco, J. G., \& López Arellano, O. (1997). Condiciones de vida en las ciudades, políticas públicas y acciones privadas en el campo de la salud. Diseño y Sociedad, 21-28. Obtenido de

http://148.206.107.15/biblioteca_digital/estadistica.php?id_host=11\&tipo=ARTICU LO\&id $=4773 \&$ archivo $=11-293-$

4773uak.pdf\&titulo $=$ Condiciones $\% 20 \mathrm{de} \% 20$ vida $\% 20$ en $\% 201$ as $\% 20$ ciudades, $\% 20$ pol $\%$ C3\%ADticas $\% 20 \mathrm{p} \% \mathrm{C} 3 \%$ BAblicas $\% 20 \mathrm{y} \% 20$ acciones $\% 20$ privadas $\% 20$ en $\% 20 \mathrm{el} \% 20 \mathrm{ca}$ $\mathrm{mpo} \% 20 \mathrm{de} \% 20$ 
Comagni, R. (2005). Economia Urbana. Barcelona: Antonio Bosch.

Comagni, R. (2005). Economia Urbana. Barcelona España: Antonio Bosch.

Dockendorff , \& Rodríguez. (1996). Política de desarrollo territorial. Versión preliminar para discusión. En Chile Urbano. Antecedentes de la consulta nacional para la formulación de una nueva política de desarrollo urbano 1993-1996. Santiago de Chile: MINVU .

Dorsch, F. (1995). Diccionario de psicología. Barcelona: Edit Herder .

Francis, J., Giles-Corti, B., Wood, L., \& Knuiman, M. (2012). Creating sense of community: The role of public space. Journal of Environmental Psychology, 32, 401-409.

Fried, M., \& Gleicher, P. (1961). Some Sources of Residential Satisfaction in Urban Slum. Journal of American Institute of Planners, 27, 305-315.

Gobierno de la República de Mexico. (20 de marzo de 2013). bttp://www.sev.gob.mx/educaciontecnologica/files/2013/05/PND_2013_2018.pdf. Obtenido de Plana Nacional de Desarrollo. República de Mexico: http://www.sev.gob.mx/educaciontecnologica/files/2013/05/PND_2013_2018.pdf

Gobierno de la Repùblico de México. (30 de abril de 2014). Programa Nacional de vivienda. Obtenido de www.dof.gob.mx: http:/ /www.dof.gob.mx/nota_detalle.php?codigo $=5342865 \&$ fecha $=30 / 04 / 2014$

Hombrados Mendieta, M. I. (2011). Sentido de comunidad. En I. Fernández, J. F. Morales, \& F. Molero, Psicología de la intervención comunitaria (págs. 97-126). Madrid: Desclée de Brower DDB.

Maya Jariego, I. (2004). Sentido de comunidad y potenciación comunitaria. Apuntes de Psicología, 22(2), 187-211. 